\title{
Análisis de Regresión y Correlación en la Prueba SABER 11 en el período
} 2011 al 2017

\section{Analysis of Regression and Correlation in the SABER 11 Test in the period 2011 to 2017}

Jorge Enrique Díaz-Pinzón.

Universidad Nacional de Colombia.

jediazp@unal.edu.co

\section{RESUMEN.}

Se realizó un estudio de investigación relacionado con los puntajes obtenidos por los estudiantes de grado once de la Institución Educativa General Santander del municipio Soacha-Cundinamarca de la jornada tarde, en la prueba SABER 11 entre los años 2011 y 2017 con el objetivo de comparar los resultados obtenidos. A partir del análisis estadístico de los datos aplicando la ANOVA, se observó que hay correlación entre tiempo y puntaje $(R=, 780)$, tiempo-matemáticas $(R=, 748)$, tiempo-inglés $(R=, 933)$, tiempo-lectura crítica $(R=, 945)$, el cual fue positivo es decir que existen una relaciones directamente proporcionales entre las variables.

\section{PALABRAS CLAVE.}

Educación, Calidad de la Educación, Evaluación comparativa, Educación comparada, Correlación

\section{ABSTRACT.}

A research study was carried out related to the scores obtained by the eleventh grade students of the General Santander Educational Institution of the Soacha-Cundinamarca municipality of the afternoon session, in the SABER 11 test between 2011 and 2017 with the aim of comparing the results obtained From the statistical analysis of the data applying the ANOVA, it was observed that there is a correlation between time and score $(R=, 780)$, timemathematics $(R=, 748)$, time-English $(R=, 933)$, time- critical reading $(R=, 945)$, which was positive is to say that there are directly proportional relationships between the variables.

\section{KEY WORDS.}

Education, Quality of Education, Comparative evaluation, Comparative education, Correlation.

\section{Introducción.}

De acuerdo con el Decreto 869 (2010). El Examen de Estado de la Educación Media, ICFESSABER $11^{\circ}$, que aplica el Instituto Colombiano para la Evaluación de la Educación (ICFES) es un instrumento estandarizado para la evaluación externa, que conjuntamente con los exámenes que se aplican en los grados $5^{\circ}, 9^{\circ}$ y al finalizar el pregrado, hace parte de los instrumentos que conforman el Sistema Nacional de Evaluación.

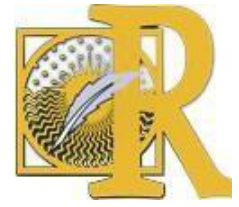


Según el (Congreso de la República de Colombia, 2009), la Ley 1324 de 2009 le confiere al Instituto Colombiano para Evaluación de la Educación (ICFES) la misión de evaluar, mediante exámenes externos estandarizados, la formación que se ofrece en el servicio educativo en los distintos niveles. También establece que el Ministerio de Educación Nacional (MEN) define lo que debe evaluarse en estos exámenes.

Según el (MENa,2010), la prueba SABER $11^{\circ}$ evaluación del nivel de la Educación Media a partir del año 2014 se ordena con las evaluaciones de la Educación Básica para suministrar información a la comunidad educativa en el progreso de las competencias básicas que debe desarrollar un estudiante durante el paso por la vida escolar. Conjuntamente de ser un instrumento que retroalimenta al Sistema Educativo la prueba SABER 11.

\section{Pruebas Pisa.}

Según el (MENb, 2006), es un programa de la Organization for Economic Cooperation and Development (OECD) que se realiza en 58 países, valora conocimientos y habilidades para la vida, coherentes con los dominios de comprensión lectora, matemática y científica. Son pruebas estandarizadas, encaminadas a jóvenes de 15 años que estén cursando al menos grado $7^{\circ}$. En Colombia, la prueba piloto se aplicó en 2005 a 1.720 estudiantes de 55 instituciones escolares, y a 5.250 estudiantes de 150 instituciones, en 2006.

\section{Estudio regional comparativo y explicativo (ERCE).}

De acuerdo con (ICFES, 2017), otra prueba que encontramos es El Estudio Regional Comparativo y Explicativo (ERCE) es el estudio educativo a gran escala más significativo de Latinoamérica. Este estudio evalúa el aprendizaje de los estudiantes en diferentes áreas e identifica elementos asociados al mismo, formando uso de pruebas estandarizadas y cuestionarios de contexto. Esta versión del estudio evaluará las competencias en lenguaje, matemáticas y ciencias dentro del ciclo de educación básica (primaria y secundaria) y ofrecerá un diagnóstico para cada país participante. De esta forma, para Colombia, participar de ERCE 2019 aportará información sobre la calidad educativa en el país, la cual puede orientar la revisión de prácticas educativas y la toma de decisiones sobre políticas públicas que optimicen nuestro sistema educativo.

\section{Primer Estudio Regional Comparativo y Explicativo (PERCE).}

Para el (ICFES, 2017), el Primer Estudio Regional Comparativo y Explicativo valoró lectura y matemática y analizó por factores asociados en los grados tercero y cuarto de educación primaria. Los informes suministran información sobre el desempeño de los estudiantes, materia para que los profesores optimicen sus prácticas de enseñanza.

\section{Segundo Estudio Regional Comparativo y Explicativo (SERCE).}

De acuerdo con (ICFES, 2017), el Segundo Estudio Regional Comparativo y Explicativo evalúa y coteja el desempeño obtenido por estudiantes latinoamericanos de tercero y sexto grados en las áreas de lenguaje, matemáticas y ciencias de la naturaleza, y busca enfocarlo a partir de distintos componentes escolares y de contexto, con el fin de crear conocimiento relevante para la toma de decisiones de política educativa, mejorar las prácticas docentes y

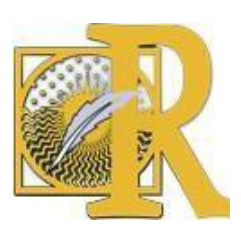


escolares, y suscitar una mayor equidad en la distribución del aprendizaje en los sistemas educativos de la región.

\section{Tercer Estudio Regional Comparativo y Explicativo (TERCE).}

El objetivo principal del Tercer Estudio Regional Comparativo y Explicativo (TERCE) es conseguir datos comparativos sobre del cometido escolar de los estudiantes de grados tercero y sexto de América Latina y el Caribe en las áreas de Ciencias, Matemáticas y Lectura, así como examinar los factores asociados a la práctica escolar para retroalimentar la política educativa. (ICFES, 2017)

\section{Prueba TIMSS.}

Lo describe el (MENb, 2006), de la International Association for the Evaluation of Educational Achievement (IEA), suministra información confiable y pertinente sobre el beneficio académico de estudiantes de Estados Unidos de grados $4^{\circ}$ y $8^{\circ}$, en Matemáticas y Ciencias Naturales, y lo confronta con el de otros países (participan 59).

\section{Población.}

Esta investigación se centró en los resultados de los estudiantes de las jornada y tarde de le institución educativa General Santander del municipio de Soacha-Cundinamarca, con la información de los resultados agregados de la prueba Saber 11-2 para los años 2011 al 2017.

\section{Hipótesis}

La hipótesis del presente trabajo de investigación se diseña como una relación causal y se enuncia de la siguiente forma:

Hipótesis Alterna (Ha): Existe correlación entre las variables tiempo y puntaje, para las asignaturas, matemáticas, inglés y lectura crítica con los resultados de la prueba SABER 11, para los años 2011 al 2017.

Hipótesis Nula (Ho): No existe correlación entre las variables tiempo y puntaje, para las asignaturas, matemáticas, inglés y lectura crítica con los resultados de la prueba SABER 11, para los años 2011 al 2017.Prueba Estadística

Se llevó a cabo una prueba de coeficiente de correlación de Pearson, que es un índice estadístico que evalúa la relación lineal entre dos variables cuantitativas. A diferencia de la covarianza, la correlación de Pearson es independiente de la escala de medida de las variables (Parisca, 2009). 
El valor del índice de correlación varía en el intervalo $[-1,+1]$.

Interpretación:

Si $r=0$ No existe correlación entre las variables

Si $0.00 \leq r \pm 0.20$ existe correlación no significativa

Si $\pm 0.20 \leq r< \pm 0.40$ existe una correlación baja

Si $\pm 0.40 \leq r< \pm 0.70$ existe una correlación significativa

Si $\pm 0.70 \leq r< \pm 1.00$ existe un alto grado de correlación

Si $r=1$ existe una correlación perfecta positiva

Si $r=-1$ existe una correlación perfecta negativa

En el análisis estadístico se incluirá lo siguiente:

El valor de significancia de la prueba es de $\alpha=0.05(5 \%)$, si es $>$ se acepta la hipótesis nula si es < se rechaza la hipótesis nula. Se utilizó el Software SPSS v 23.0.

\section{Resultados.}

En la presente investigación se procedió a trabajar con los resultados de los puntajes de estudiantes de grado once en la prueba SABER 11, para las asignaturas, matemáticas, inglés y lectura crítica, para los años 2011 al 2017.

La aplicación de una prueba estadística nos admite comprobar si la correlación observada en la muestra es estadísticamente significativa. En la tabla 1 se observa que el valor $p$ resultante es inferior al nivel de significación establecido $(p<0,05)$, afirmamos, con un riesgo $p$ de equivocarnos, que $\mathrm{R}$ es distinto de 0 en la población. La sig. (Significancia) es $0,035 \leq 0,05$ se acepta $\mathrm{Ha}$, es decir que si hay relación entre las variables. Podemos observar que la correlación, 788 es positiva, es decir que existe una relación directamente proporcional entre variables tiempo (años) y puntaje.

Tabla 1. Correlaciones año-puntaje.

\begin{tabular}{|ll|r|r|}
\hline & & Año & \multicolumn{2}{|c|}{ Puntaje } \\
\hline \multirow{4}{*}{ Año } & Correlación de Pearson & 1 &, $788^{*}$ \\
& Sig. (bilateral) & &, 035 \\
& $N$ & 7 & 7 \\
Puntaje & Correlación de Pearson &, $788^{*}$ & 1 \\
& Sig. (bilateral) &, 035 & 7 \\
\hline
\end{tabular}

\section{Coeficiente de correlación múltiple $\mathbf{R}$}

La primera información que se obtiene en la tabla 2, se refiere al coeficiente de correlación múltiple R. Puesto que sólo tenemos dos variables, el coeficiente de correlación múltiple no es otra cosa que el valor absoluto del coeficiente de correlación de Pearson entre esas dos variables.

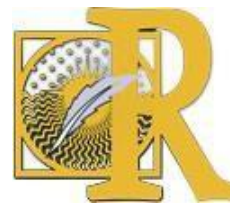




\section{Bondad de ajuste.}

Una vez ajustada la recta de regresión a la nube de observaciones es transcendental disponer de una medida que evalúe la bondad del ajuste realizado y que admita decidir si el ajuste lineal es suficiente, como medida de bondad se utiliza el coeficiente de correlación.

Tabla 2. Resumen del Modelo.

\begin{tabular}{|c|c|c|c|}
\hline Modelo & $\mathrm{R}$ & $\mathrm{R}$ cuadrado & $\mathrm{R}$ cuadrado corregida \\
\hline 1 &, $780^{\mathrm{a}}$ &, 621 &, 545 \\
\hline
\end{tabular}

En la tabla 2, se observa que la correlación entre las variables fue de ,780 la cual es directamente proporcional entre sus variables, el valor $\mathrm{R}^{2}$ es de ,621 que corresponde a la varianza explicada del modelo es de un $62,1 \%$, he indica que la variación en el puntaje esta explicada por el tiempo (años) que los estudiantes obtuvieron en la prueba SABER 11, entre el período 2001 al 2017.

En la tabla 3 se observa que el valor $p$ resultante es inferior al nivel de significación establecido $(p<0,05$ ), afirmamos, con un riesgo $p$ de equivocarnos, que $R$ es distinto de 0 en la población. La sig. (Significancia) es $0,05 \leq 0,05$ se acepta $\mathrm{Ha}$, es decir que si hay relación entre las variables. Podemos observar que la correlación ,748 es positiva, es decir que existe una relación directamente proporcional entre variables tiempo (años) y la prueba de matemáticas.

Tabla 3. Correlaciones año- matemáticas

\begin{tabular}{|ll|r|r|}
\hline & & \multicolumn{1}{|c|}{ Año } & \multicolumn{2}{|c|}{ Matemáticas } \\
\hline \multirow{3}{*}{ Año } & Correlación de Pearson & 1 &, 748 \\
& Sig. (bilateral) & &, 05 \\
& $\mathrm{~N}$ & 7 & 7 \\
Matemáticas & Correlación de Pearson &, 748 & 1 \\
& Sig. (bilateral) &, 053 & 7 \\
\hline
\end{tabular}

\section{Bondad de ajuste.}

Una vez ajustada la recta de regresión a la nube de observaciones es transcendental disponer de una medida que evalúe la bondad del ajuste realizado y que admita decidir si el ajuste lineal es suficiente, como medida de bondad se utiliza el coeficiente de correlación.

Tabla 4. Resumen del Modelo.

\begin{tabular}{|c|c|c|c|}
\hline Modelo & $\mathrm{R}$ & R cuadrado & R cuadrado corregida \\
\hline 1 &, $748^{\mathrm{a}}$ &, 560 &, 472 \\
\hline
\end{tabular}


En la tabla 4, se observa que la correlación entre las variables fue de ,748 la cual es directamente proporcional entre sus variables, el valor $\mathrm{R}^{2}$ es de ,560 que corresponde a la varianza explicada del modelo es de un $56,0 \%$, he indica que la variación en el puntaje en matemáticas esta explicada por el tiempo (años) que los estudiantes obtuvieron en la prueba SABER 11, entre el período 2001 al 2017.

En la tabla 5, se observa que el valor $\mathrm{p}$ resultante es inferior al nivel de significación establecido $(p<0,05)$, afirmamos, con un riesgo $p$ de equivocarnos, que $R$ es distinto de 0 en la población. La sig. (Significancia) es 0,002 $\leq 0,05$ se acepta Ha, es decir que si hay relación entre las variables. Podemos observar que la correlación ,933 es positiva, es decir que existe una relación directamente proporcional entre variables tiempo (años) y puntaje en inglés.

Tabla 5. Correlaciones año-Inglés.

\begin{tabular}{|ll|r|r|}
\hline & & \multicolumn{1}{|c|}{ Año } & \multicolumn{1}{|c|}{ Inglés } \\
\hline \multirow{4}{*}{ Año } & Correlación de & 1 &, $933^{* *}$ \\
& Pearson & &, 002 \\
& Sig. (bilateral) & 7 & 7 \\
& $N$ &, $933^{* *}$ & 1 \\
Inglés & Correlación de &, 002 & \\
& Pearson & 7 & 7 \\
\hline
\end{tabular}

**. La correlación es significativa al nivel 0,01 (bilateral).

\section{Bondad de ajuste.}

Una vez ajustada la recta de regresión a la nube de observaciones es transcendental disponer de una medida que evalúe la bondad del ajuste realizado y que admita decidir si el ajuste lineal es suficiente, como medida de bondad se utiliza el coeficiente de correlación.

Tabla 6. Resumen del Modelo.

\begin{tabular}{|c|c|c|c|}
\hline Modelo & $\mathrm{R}$ & $\mathrm{R}$ cuadrado & R cuadrado corregida \\
\hline 1 &, $933^{\mathrm{a}}$ &, 871 &, 845 \\
\hline
\end{tabular}

En la tabla 6, se observa que la correlación entre las variables fue de ,933 la cual es directamente proporcional entre sus variables, el valor $\mathrm{R}^{2}$ es de, 871 que corresponde a la varianza explicada del modelo es de un $87,1 \%$, he indica que la variación en el puntaje en inglés esta explicada por el tiempo (años) que los estudiantes obtuvieron en la prueba SABER 11, entre el período 2001 al 2017. 
En la tabla 7, se aprecia que el valor $p$ resultante es inferior al nivel de significación establecido $(p<0,05$ ), afirmamos, con un riesgo $p$ de equivocarnos, que $R$ es distinto de 0 en la población. La sig. (Significancia) es 0,001 $\leq 0,05$ se acepta $\mathrm{Ha}$, es decir que si hay relación entre las variables. Podemos observar que la correlación ,945 es positiva, es decir que existe una relación directamente proporcional entre variables tiempo (años) y puntaje en inglés.

Tabla 7. Correlaciones. Año-Lectura crítica.

\begin{tabular}{|ll|r|r|}
\hline & & \multicolumn{1}{|c|}{ Año } & Lectura crítica \\
\hline \multirow{4}{*}{ Año } & Correlación de & 1 &, $945^{* *}$ \\
& Pearson & &, 001 \\
& Sig. (bilateral) & 7 & 7 \\
& $\mathrm{~N}$ &, $945^{* *}$ & 1 \\
& Correlación de &, 001 & \\
Lectura & Pearson & 7 & 7 \\
& Sig. (bilateral) & $\mathrm{N}$ & \\
& & &
\end{tabular}

**. La correlación es significativa al nivel 0,01 (bilateral).

\section{Bondad de ajuste.}

Una vez ajustada la recta de regresión a la nube de observaciones es transcendental disponer de una medida que evalúe la bondad del ajuste realizado y que admita decidir si el ajuste lineal es suficiente, como medida de bondad se utiliza el coeficiente de correlación.

Tabla 8. Resumen del Modelo.

\begin{tabular}{|c|c|c|c|}
\hline Modelo & $\mathrm{R}$ & $\mathrm{R}$ cuadrado & R cuadrado corregida \\
\hline 1 &, $945^{\mathrm{a}}$ &, 893 &, 872 \\
\hline
\end{tabular}

En la tabla 8, se observa que la correlación entre las variables fue de ,933 la cual es directamente proporcional entre sus variables, el valor $\mathrm{R}^{2}$ es de, 893 que corresponde a la varianza explicada del modelo es de un $89,3 \%$, he indica que la variación en el puntaje en lectura críticas esta explicada por el tiempo (años) que los estudiantes obtuvieron en la prueba SABER 11, entre el período 2001 al 2017. 


\section{Regresión Lineal.}

Figura 1. Diagrama de dispersión y recta de regresión. Tiempo-puntaje.

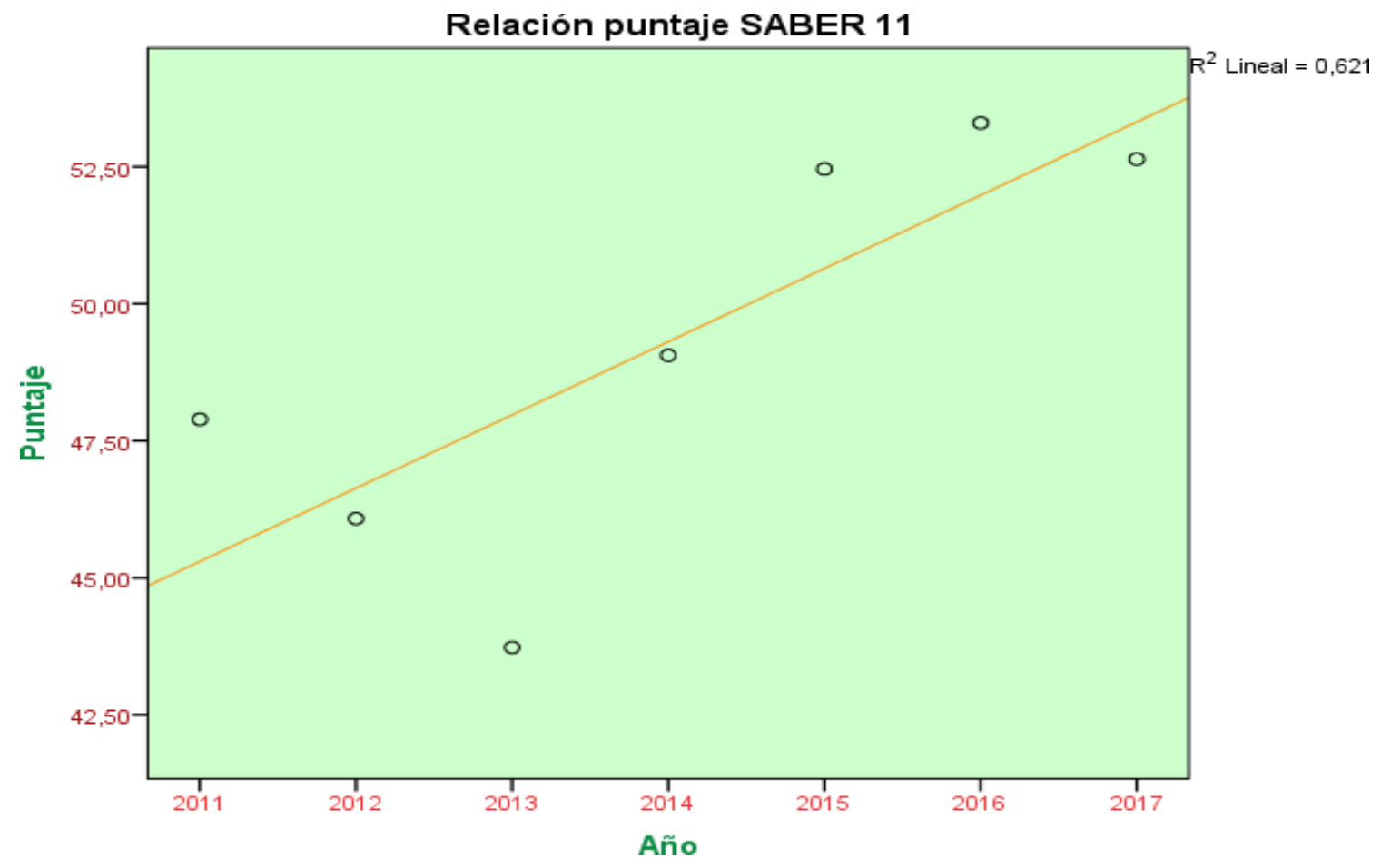

En el eje vertical podemos observar el puntaje obtenido y en el eje horizontal el tiempo en años. A simple vista, se puede observar que existe una relación positiva entre ambas variables: conforme aumenta el tiempo, también aumenta el puntaje.

Tabla 9. ANOVAa

\begin{tabular}{|c|c|c|c|c|c|c|}
\hline \multicolumn{2}{|c|}{ Modelo } & $\begin{array}{c}\text { Suma de } \\
\text { cuadrados }\end{array}$ & gl & $\begin{array}{c}\text { Media } \\
\text { cuadrática }\end{array}$ & $F$ & Sig. \\
\hline \multirow{3}{*}{1} & Regresión & 50,009 & 1 & 50,009 & 8,179 &, $035^{b}$ \\
\hline & Residual & 30,571 & 5 & 6,114 & & \\
\hline & Total & 80,580 & 6 & & & \\
\hline
\end{tabular}

a. Variable dependiente: Puntaje

b. Variables predictoras: (Constante), Año

En la tabla 9 del ANOVA, nos muestra si existe o no relación significativa entre las variables. El nivel crítico (Sig.) nos indica que, si hay un supuesto que el valor poblacional de $R$ es cero, es poco probable (probabilidad $=0,035$ ) que $R$, en esta muestra, tome el valor ,780. Lo cual demuestra que $\mathrm{R}$ es mayor que cero y que, por ende, ambas variables están linealmente correlacionadas. 
Tabla 10. Coeficientes ${ }^{a}$

\begin{tabular}{|c|c|c|c|c|c|c|}
\hline \multirow{2}{*}{\multicolumn{2}{|c|}{ Modelo }} & \multicolumn{2}{|c|}{ Coeficientes no estandarizados } & Coeficientes & \multirow[t]{2}{*}{$\mathrm{t}$} & \multirow[t]{2}{*}{ Sig. } \\
\hline & & B & Error típ. & Beta & & \\
\hline \multirow[t]{2}{*}{1} & (Constante) & $-2642,259$ & 941,125 & & $-2,808$ & ,038 \\
\hline & Año & 1,336 & ,467 & ,788 & 2,860 & 035 \\
\hline
\end{tabular}

a. Variable dependiente: Puntaje

En la tabla 10, se observan los coeficientes obtenidos a partir de la regresión lineal, podemos analizar que los valores de significancia de la variable independiente tiempo, es de 0,038 esto indica que es significativo, de esta manera pertenece a la ecuación. Lo cual podemos realizar un pronóstico de puntaje obtenido según el tiempo, como se observa en la siguiente ecuación:

\section{$Y=-2642,259+1,336 X$}

Los coeficientes $-2642,259$ y 1,336 definen la recta. El coeficiente 1,336 es la pendiente de la recta; el cambio medio que se obtiene en el puntaje $(Y)$ por cada unidad de cambio que se produce de tiempo en minutos $(X)$ le corresponde un incremento de -2642,259 puntaje $(Y)$

Figura 2. Diagrama de dispersión y recta de regresión. Tiempo-Matemáticas.

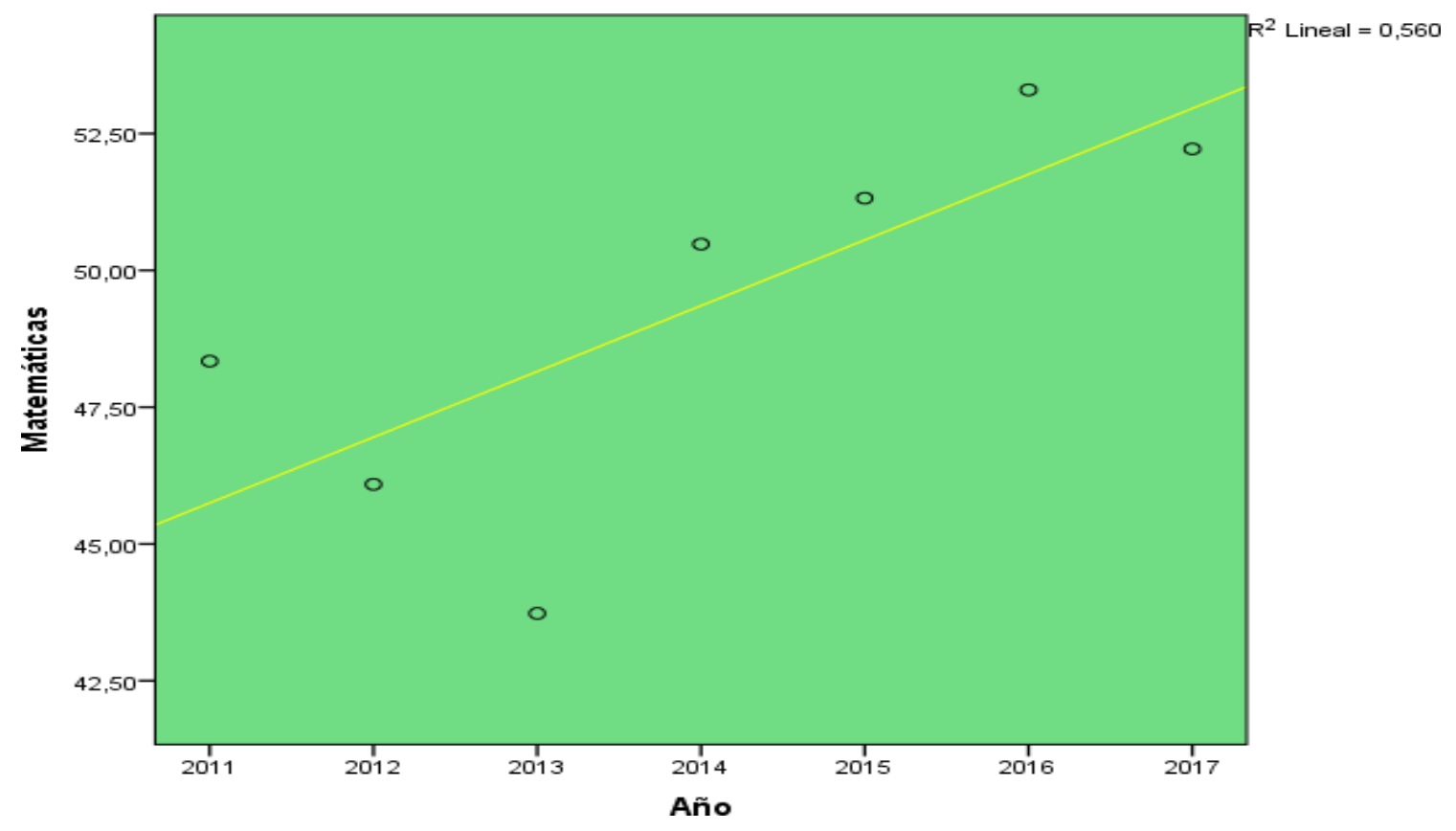


En el eje vertical podemos observar el puntaje obtenido en matemáticas y en el eje horizontal el tiempo en años. A simple vista, se puede observar que existe una relación positiva entre ambas variables: conforme aumenta el tiempo, también aumenta el puntaje en matemáticas.

Tabla 11.ANOVAa

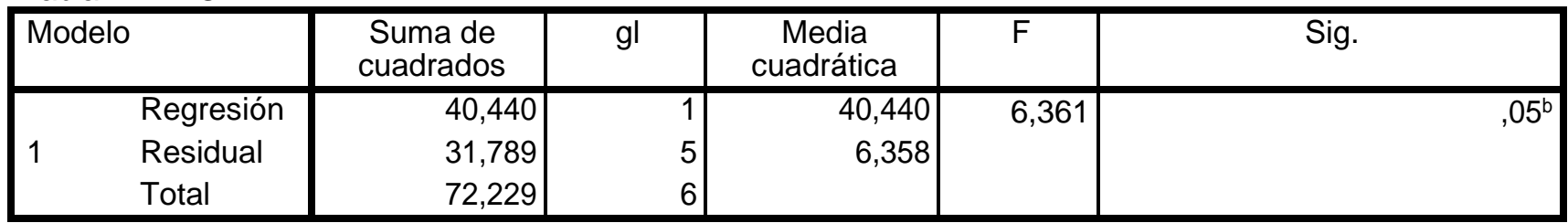

a. Variable dependiente: Matemáticas.

b. Variables predictoras: (Constante), Año.

En la tabla 11 del ANOVA, nos muestra si existe o no relación significativa entre las variables. El nivel crítico (Sig.) nos indica que, si hay un supuesto que el valor poblacional de $R$ es cero, es poco probable (probabilidad $=0,05$ ) que $R$, en esta muestra, tome el valor ,748. Lo cual demuestra que $\mathrm{R}$ es mayor que cero y que, por ende, ambas variables están linealmente correlacionadas.

\begin{tabular}{|c|c|c|c|c|c|}
\hline \multirow[t]{2}{*}{ Modelo } & \multicolumn{2}{|c|}{$\begin{array}{l}\text { Coeficientes no } \\
\text { estandarizados }\end{array}$} & $\begin{array}{l}\text { Coeficientes } \\
\text { tipificados }\end{array}$ & \multirow[t]{2}{*}{$\mathrm{t}$} & \multirow[t]{2}{*}{ Sig. } \\
\hline & \begin{tabular}{l|l}
$\mathrm{B}$ &
\end{tabular} & Error típ. & Beta & & \\
\hline $\begin{array}{ll}1 & \text { (Constante) } \\
\text { Año }\end{array}$ & $\begin{array}{r}-2371,042 \\
1,202\end{array}$ & $\begin{array}{r}959,698 \\
, 477\end{array}$ & ,748 & $\begin{array}{r}-2,471 \\
2,522\end{array}$ & $\begin{array}{r}, 05 \\
, 053\end{array}$ \\
\hline
\end{tabular}

a. Variable dependiente: Matemáticas.

En la tabla 12, se observan los coeficientes obtenidos a partir de la regresión lineal, podemos analizar que los valores de significancia de la variable independiente tiempo, es de 0,05 esto indica que es significativo, de esta manera pertenece a la ecuación. Lo cual podemos realizar un pronóstico de puntaje obtenido según el tiempo, como se observa en la siguiente ecuación:

\section{$Y=-2371,042+1,202 X$}

Los coeficientes $-2371,042$ y 1,202 definen la recta. El coeficiente 1,202 es la pendiente de la recta; el cambio medio que se obtiene en el puntaje $(Y)$ por cada unidad de cambio que se produce de tiempo en minutos $(X)$ le corresponde un incremento de $-2371,042$ puntaje $(\mathrm{Y})$. 
Figura 3. Diagrama de dispersión y recta de regresión. Tiempo-Inglés.

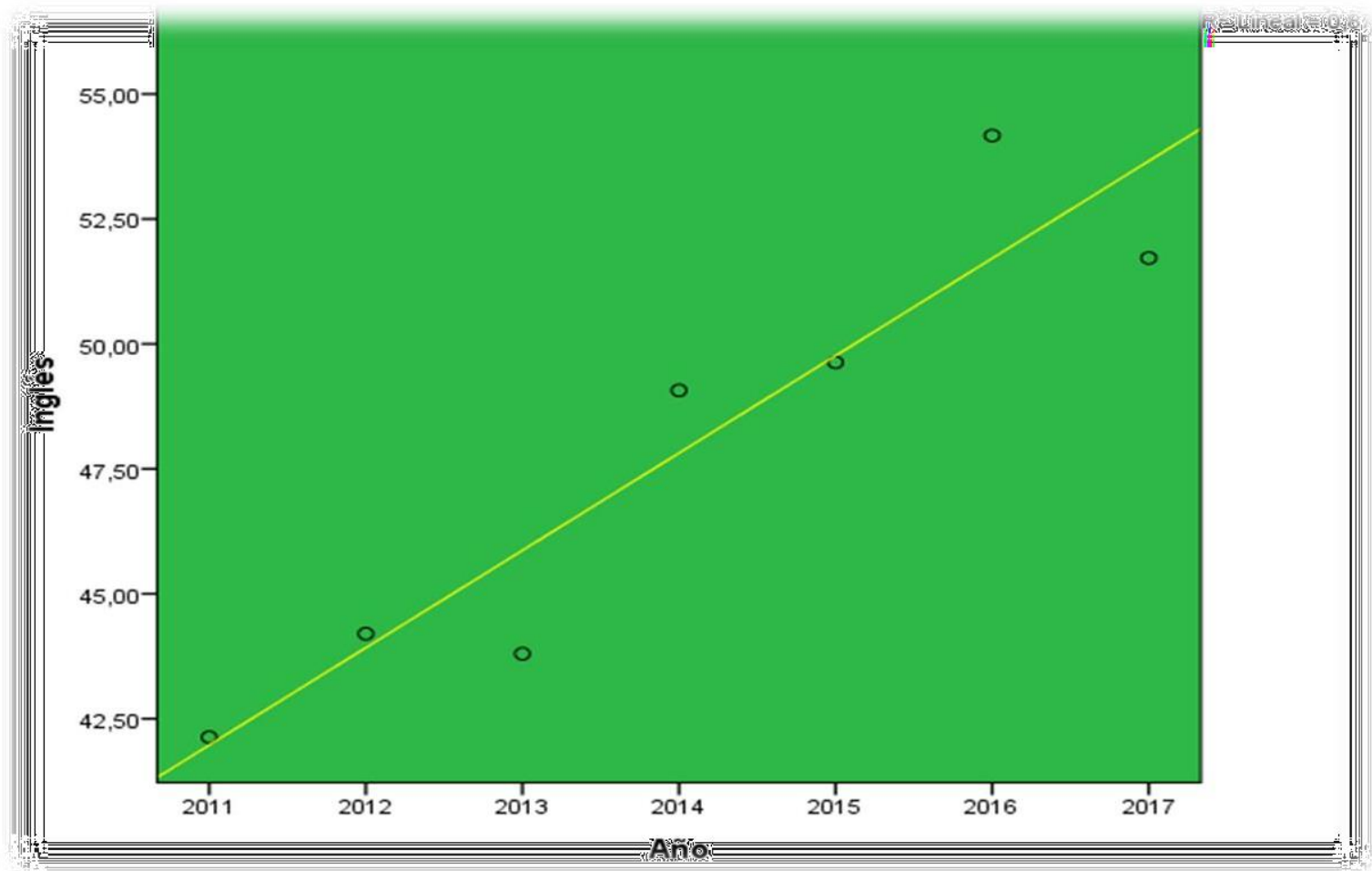

En la figura 3, en el eje vertical podemos observar el puntaje obtenido en inglés y en el eje horizontal el tiempo en años. A simple vista, se puede observar que existe una relación positiva entre ambas variables: conforme aumenta el tiempo, también aumenta el puntaje en inglés.

Tabla 13. ANOVAa

\begin{tabular}{|c|c|c|c|c|c|c|}
\hline \multicolumn{2}{|c|}{ Modelo } & $\begin{array}{l}\text { Suma de } \\
\text { cuadrados }\end{array}$ & gl & $\begin{array}{c}\text { Media } \\
\text { cuadrática }\end{array}$ & $\mathrm{F}$ & Sig. \\
\hline \multirow{3}{*}{1} & Regresión & 106,236 & 1 & 106,236 & 33,671 &, $002^{b}$ \\
\hline & Residual & 15,776 & 5 & 3,155 & & \\
\hline & Total & 122,012 & 6 & & & \\
\hline
\end{tabular}

a. Variable dependiente: Inglés.

b. Variables predictoras: (Constante), Año.

En la tabla 13 del ANOVA, nos muestra si existe o no relación significativa entre las variables. El nivel crítico (Sig.) nos indica que, si hay un supuesto que el valor poblacional de $R$ es cero, es poco probable (probabilidad $=0,02$ ) que $R$, en esta muestra, tome el valor ,933. Lo cual demuestra que $R$ es mayor que cero y que, por ende, ambas variables están linealmente correlacionadas. 
Tabla 14. Coeficientes ${ }^{a}$

\begin{tabular}{|c|c|c|c|c|c|}
\hline \multirow[t]{2}{*}{ Modelo } & \multicolumn{2}{|c|}{$\begin{array}{l}\text { Coeficientes no } \\
\text { estandarizados }\end{array}$} & $\begin{array}{c}\text { Coeficientes } \\
\text { tipificados }\end{array}$ & \multirow[t]{2}{*}{$\mathrm{t}$} & \multirow[t]{2}{*}{ Sig. } \\
\hline & $B$ & Error típ. & Beta & & \\
\hline $\begin{array}{l}\text { (Constante) } \\
\text { Año }\end{array}$ & $\begin{array}{r}-3875,167 \\
1,948 \\
\end{array}$ & $\begin{array}{r}676,070 \\
, 336 \\
\end{array}$ & ,933 & $\begin{array}{r}-5,732 \\
5,803\end{array}$ & $\begin{array}{l}, 002 \\
, 002\end{array}$ \\
\hline
\end{tabular}

a. Variable dependiente: Inglés

En la tabla 14, se observan los coeficientes obtenidos a partir de la regresión lineal, podemos analizar que los valores de significancia de la variable independiente tiempo, es de 0,05 esto indica que es significativo, de esta manera pertenece a la ecuación. Lo cual podemos realizar un pronóstico de puntaje obtenido según el tiempo, como se observa en la siguiente ecuación:

\section{$Y=-3875,167+1,948 X$}

Los coeficientes $-3875,167$ y 1,948 definen la recta. El coeficiente 1,948 es la pendiente de la recta; el cambio medio que se obtiene en el puntaje $(Y)$ por cada unidad de cambio que se produce de tiempo en minutos $(X)$ le corresponde un incremento de $-3875,167$ puntaje $(\mathrm{Y})$.

Figura 4. Diagrama de dispersión y recta de regresión. Tiempo-Lectura Crítica.

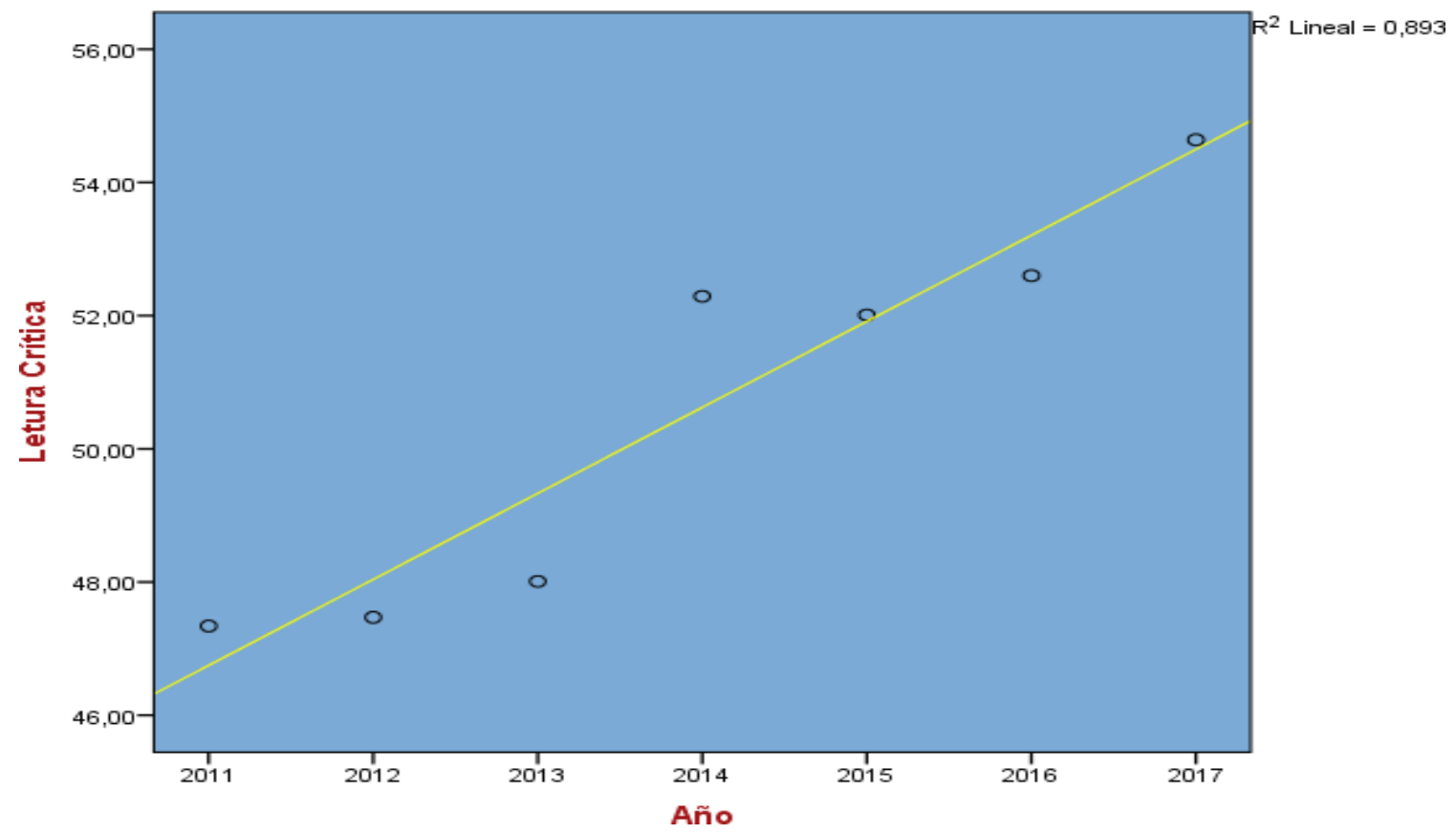


En la figura 4, en el eje vertical podemos observar el puntaje obtenido en lectura crítica y en el eje horizontal el tiempo en años. A simple vista, se puede observar que existe una relación positiva entre ambas variables: conforme aumenta el tiempo, también aumenta el puntaje en lectura crítica.

Tabla 15. ANOVAa

\begin{tabular}{|c|c|c|c|c|c|c|}
\hline \multicolumn{2}{|c|}{ Modelo } & $\begin{array}{l}\text { Suma de } \\
\text { cuadrados }\end{array}$ & gl & $\begin{array}{c}\text { Media } \\
\text { cuadrática }\end{array}$ & $\mathrm{F}$ & Sig. \\
\hline \multirow{3}{*}{1} & Regresión & 46,698 & 1 & \multirow{3}{*}{$\begin{array}{r}46,698 \\
1,119\end{array}$} & \multirow{3}{*}{41,719} & \multirow{3}{*}{, $001^{b}$} \\
\hline & Residual & 5,597 & 5 & & & \\
\hline & Total & 52,295 & 6 & & & \\
\hline
\end{tabular}

a. Variable dependiente: Lectura Crítica.

b. Variables predictoras: (Constante), Año.

En la tabla 15 del ANOVA, nos muestra si existe o no relación significativa entre las variables. El nivel crítico (Sig.) nos indica que, si hay un supuesto que el valor poblacional de $R$ es cero, es poco probable (probabilidad $=0,01$ ) que $R$, en esta muestra, tome el valor ,945. Lo cual demuestra que $\mathrm{R}$ es mayor que cero y que, por ende, ambas variables están linealmente correlacionadas.

\begin{tabular}{|c|c|c|c|c|c|}
\hline \multirow[t]{2}{*}{ Modelo } & \multicolumn{2}{|c|}{$\begin{array}{l}\text { Coeficientes no } \\
\text { estandarizados }\end{array}$} & $\begin{array}{c}\text { Coeficientes } \\
\text { tipificados }\end{array}$ & \multirow[t]{2}{*}{$t$} & \multirow[t]{2}{*}{ Sig. } \\
\hline & \begin{tabular}{l|l}
$\mathrm{B}$ \\
\end{tabular} & Error típ. & Beta & & \\
\hline $\begin{array}{l}\text { (Constante) } \\
\text { Año }\end{array}$ & $\begin{array}{r}-2550,314 \\
1,291\end{array}$ & $\begin{array}{r}402,681 \\
, 200\end{array}$ & 945, & $\begin{array}{r}-6,333 \\
6,459\end{array}$ & $\begin{array}{l}, 001 \\
, 001\end{array}$ \\
\hline
\end{tabular}

a. Variable dependiente: Lectura Crítica.

En la tabla 16, se observan los coeficientes obtenidos a partir de la regresión lineal, podemos analizar que los valores de significancia de la variable independiente tiempo, es de 0,05 esto indica que es significativo, de esta manera pertenece a la ecuación. Lo cual podemos realizar un pronóstico de puntaje obtenido según el tiempo, como se observa en la siguiente ecuación:

\section{$Y=-2550,314+1,291 X$}

Los coeficientes $-2550,314$ y 1,291 definen la recta. El coeficiente 1,291 es la pendiente de la recta; el cambio medio que se obtiene en el puntaje $(\mathrm{Y})$ por cada unidad de cambio que se produce de tiempo en minutos $(X)$ le corresponde un incremento de $-2550,314$ puntaje $(\mathrm{Y})$. 


\section{Conclusiones.}

Con respecto a la hipótesis sobre la existencia de la correlación entre las variables tiempo (años) y puntaje obtenidos demostraron que existen relaciones directamente proporcionales entre las variables.

Se determinó la correlación entre las variables fue de ,780 la cual es directamente proporcional entre sus variables, el valor $\mathrm{R}^{2}$ es de ,621 que corresponde a la varianza explicada del modelo es de un $62,1 \%$, para las variables tiempo - puntaje; ahora para la correlación entre las variables tiempo-matemáticas fue de ,748 la cual es directamente proporcional entre sus variables, el valor $\mathrm{R}^{2}$ es de ,560 que corresponde a la varianza explicada del modelo es de un $56,10 \%$, la correlación entre las variables tiempo-inglés fue de ,933 la cual es directamente proporcional entre sus variables, el valor $\mathrm{R}^{2}$ es de ,871 que corresponde a la varianza explicada del modelo es de un $87,1 \%$, para las variables tiempo inglés; ahora para la correlación entre las variables tiempo-lectura crítica fue de ,945 la cual es directamente proporcional entre sus variables, el valor $\mathrm{R}^{2}$ es de, 893 que corresponde a la varianza explicada del modelo es de un $89,3 \%$ he indica que la variación en el puntaje esta explicada por el tiempo que los estudiantes que presentaron la prueba SABER 11, durante el período 2011-2017, en la institución educativa General Santander del municipio de Soacha-Cundinamarca, Colombia.

De acuerdo con (Chica, et al, 2009), en su trabajo de investigación denominado "Determinantes del rendimiento académico en Colombia: pruebas ICFES Saber 110, 2009", para las variables institucionales se alcanzó, para matemáticas y lenguaje, que los estudiantes referentes a la jornada completa tienen un mejor cometido en la prueba que los estudiantes que estudian en las demás jornadas. Este resultado es suficiente relevante desde el punto de vista de los alcances de política económica, puesto que se debe recapacitar sensatamente una estrategia de ampliación de la cobertura sin ir en menoscabo de los resultados que obtienen los bachilleres en las pruebas de estado. Así mismo, los estudiantes que tienen mayor probabilidad de situarse en el nivel bajo, son aquellos que acuden en la jornada nocturna o sabatina y dominical. Una posición crítica de la prueba Saber $11^{\circ}$, está relacionada como un examen escrito que no articula todas las áreas, implanta un valor numérico al resultado obtenido y desecha las condiciones del contexto de cada institución educativa, por tal motivo, a todas las instituciones se les mide como si tuvieran las mismas condiciones de infraestructura física, posición geográfica, recursos físicos y humanos. Desde esta perspectiva es muy difícil medir con exactitud la calidad educativa de cada plantel.

Para tratar de mejorar en la prueba SABER 11, se pueden mejorar las prácticas de aula en matemáticas, inglés y lectura crítica, ejemplo de ello tenemos los trabajos realizados por (Díaz, 2016a;; 2017a; 2017b), sobre el uso de simuladores, logrando evidenciar una mejora en la enseñanza de las matemáticas, además de esto se debe tener una formación permanente de los docentes en el conocimiento y apropiación de las tecnologías de la información y la comunicación, para de esta forma el docente pueda utilizar nuevas herramientas ofimáticas en su práctica de aula. (Díaz, 2017c; 2018). 


\section{Referencias bibliográficas.}

- Chica, S. Galvis. D. Ramírez, A. (2009). Determinantes del rendimiento académico en Colombia: pruebas ICFES Saber 11으 2009. Recuperado de: http://www.eafit.edu.co/escuelas/economiayfinanzas/publicaciones/Documents/workingpapers/Determinantes\%20del\%20rendimiento\%20acad\%C3\%A9mico\%20en\%20Colombia\%20pruebas\%2 0ICFES\%20Saber\%2011\%C2\%BA,\%202009\%20Revisado.pdf

- Congreso de la República de Colombia. (2009). Ley 1324 de 2009: por la cual se fijan parámetros y criterios para organizar el sistema de evaluación de resultados de la calidad de la educación, se dictan normas para el fomento de una cultura de la evaluación, en procura de facilitar la inspección y vigilancia del Estado y se transforma el ICFES. Diario Oficial, 13 de julio de 2009, n. 47.409. Bogotá, D. C.: Imprenta Nacional de Colombia.

- Decreto 869 (2010). Por el cual se reglamenta el Examen de Estado de la Educación Media, ICFES SABER 11․ Ministerio de Educación Nacional. Colombia.

- Díaz, J. (2016a). Aplicación PhET: estrategia de enseñanza-aprendizaje de fracciones equivalentes. Revista Criterios, 23(1). Recuperado de: http://www.umariana.edu.co/ojsditorial/index.php/criterios/article/view/1193

- Díaz, J. (2017a). Importancia de la simulación Phet en la enseñanza y aprendizaje de fracciones equivalentes. Revista Educación y Desarrollo Social, 11(1). Recuperado de: https://revistas.unimilitar.edu.co/index.php/reds/article/view/2011

- Díaz, J. (2017b) Simulador Applet Descartes: Como didáctica de enseñanza de la función cuadrática. Revista Innova Research Journal, 2(8). Recuperado de: http://www.journaluidegye.com/magazine/index.php/innova/article/view/256

- Díaz, J. (2017c). Conocimiento y apropiación de las tecnologías de la información y la comunicación (TIC) en la formación docente. Revista Actualidades Pedagógicas, 69, 121-131 Recuperado https://revistas.lasalle.edu.co/index.php/ap/article/view/4066/3198

de:

- Díaz, J. (2018). Aprendizaje de las matemáticas con el uso de la simulación. Revista Sophia, 14(1).

- Instituto Colombiano para el Fomento de la educación Superior (ICFES). (2017). LLECE (TERCE - SERCE - PERCE). Recuperado de: http://www2.icfes.gov.co/terminos-deuso/itemlist/category/348-llece-terce-serce-perce

- Ministerio de Educación Nacional (MENa). (2010). Pruebas Saber. Recuperado de: http://www.mineducacion.gov.co/1759/w3-article-244735.html

- Ministerio de Educación Nacional (MENb). (2006). Al tablero. Recuperado de: http://www.mineducacion.gov.co/1621/article-107522.html

- Parisca, M. (2009). Coeficiente de la correlación de Pearson, media, moda y mediana. Recuperado de: https://bit.ly/328eB0k 